\title{
Clinical value of elasticity imaging and contrast-enhanced ultrasound in the diagnosis of papillary thyroid microcarcinoma
}

\author{
FENGSHENG LI $^{1 *}$, JIANLEI ZHANG ${ }^{2 *}$, YUNMEI WANG ${ }^{1}$ and LIWEN LIU ${ }^{3}$ \\ ${ }^{1}$ Department of Ultrasound, Shaanxi Provincial Cancer Hospital Affiliated to Medical School, Xi'an Jiaotong University, \\ Xi'an, Shaanxi 710061; ${ }^{2}$ Department of Ultrasonography, Yan'an People's Hospital, Yan'an, Shaanxi 716000; \\ ${ }^{3}$ Department of Ultrasonography, Xijing Hospital, Fourth Military Medical University, Xi'an, Shaanxi 710054, P.R. China
}

Received September 29, 2014; Accepted April 20, 2015

DOI: $10.3892 / 01.2015 .3387$

\begin{abstract}
The present study aimed to evaluate the value of elasticity imaging and contrast-enhanced ultrasonography (CEUS) in the differential diagnosis of papillary thyroid microcarcinoma (TMC). In total, 73 patients exhibiting a total of 80 small thyroid nodules, which were difficult to diagnose using conventional ultrasonography, underwent elasticity imaging and CEUS. The diagnostic findings were subsequently clarified by intraoperative and pathological examination, and the accuracy of the 2 diagnostic methods was compared. The correct diagnostic rate of CEUS was $85 \%$ (68/80 nodules), of which 6 cases of TMC were misdiagnosed as benign lesions and 6 benign nodules were misdiagnosed as TMC. By contrast, the accuracy rate of the elasticity imaging, based on the 5-point diagnostic method, was $92.5 \%$ (74/80 nodules), of which 3 cases of TMC were misdiagnosed as benign nodules and 3 benign nodules were misdiagnosed as TMC. Furthermore, elasticity imaging in the diagnosis of TMC was determined to have sensitivity, specificity and accuracy rates of $94.0,90.0$ and $92.5 \%$, respectively, whereas the corresponding rates for CEUS were $88.0,80.0$ and $85.0 \%$, respectively. Thus, ultrasonographic elasticity imaging exhibited significant advantages in the diagnosis of TMC compared with CEUS $(\mathrm{P}<0.05)$. The use of CEUS demonstrates no evident advantage in the diagnosis of TMC; however, an elasticity score of $\geq 3$ is of high clinical value as a diagnostic criterion for TMC.
\end{abstract}

Correspondence to: Dr Fengsheng Li, Department of Ultrasound, Shaanxi Provincial Cancer Hospital Affiliated to Medical School, Xi'an Jiaotong University, 309 Yanta West Road, Xi'an, Shaanxi 710061, P.R. China

E-mail: fengshegli@126.com

${ }^{*}$ Contributed equally

Key words: ultrasonography, microbubble, thyroid neoplasms, elasticity imaging

\section{Introduction}

At present, there are $>300$ million individuals with thyroid disease worldwide (1). In particular, thyroid nodules, which are the second most common endocrine system disease, have received increasing attention $(2,3)$. Conventional ultrasonography was previously considered to be the preferred diagnostic tool for patients with thyroid disease, and has been reported as the most effective method for the clinical assessment and treatment of this disorder (4). However, with the development of high-frequency ultrasonography, the early detection of thyroid lesions has improved (5). Thus, high-frequency ultrasonography has become the preferred method for the clinical evaluation and treatment of thyroid disease (6). Accordingly, thyroid nodules identified on cervical ultrasonographic examination account for $50 \%$ of all reported thyroid nodules (7). Papillary thyroid microcarcinoma (TMC) is an early-stage subtype of thyroid carcinoma with a maximum tumor diameter of $\leq 10 \mathrm{~mm}$ and a frequency of $65-99 \%$ among all thyroid carcinomas (8). However, nodular thyroid lesions measuring $\leq 1 \mathrm{~cm}$ in diameter may possess features that are characteristic of different types of lesions, commonly resulting in an uncertain diagnosis during pre-operative conventional ultrasonography (8). Therefore, the development of novel pre-operative diagnostic methods is required.

In previous years, contrast-enhanced ultrasound (CEUS) and ultrasonic elastography (UE) have become popular research topics, and these modalities have been proposed for the diagnosis and differential diagnosis of thyroid lesions, as well as for enhancing the diagnostic confidence for such lesions (9-11). However, due to differences in the patients investigated and the evaluation indices and methods employed between studies, the reported evaluation criteria are inconsistent (12). For example, Giuseppetti et al (13) considered the enhanced contrast patterns of thyroid nodules to be closely associated with nodule size as opposed to pathological type, and proposed that nodule size had a particular influence on the UE results. This criterion may be appropriate as UE methodology is based on external pressure applied to the inspected tissues, which may result in compressive deformation, and therefore may objectively reflect tissue stiffness. To date, the majority of studies investigating thyroid nodule diagnosis using UE have focused on differentiating between benign and 
malignant nodules (14-16). Conversely, only a limited number of studies have been conducted on the use of CEUS and UE to differentiate between papillary TMC and other types of lesions. Thus, the present study proposes that these 2 novel technologies may have the potential to enhance the accuracy of benign and malignant thyroid nodule diagnosis. Accordingly, the aim of the current study was to explore the value of UE and CEUS in the differential diagnosis of benign and malignant lesions of the thyroid.

\section{Subjects and methods}

Subjects. Between May 2012 and September 2013, a retrospective analysis was performed on 73 patients that possessed a total of 80 micro-thyroid space-occupying lesions. The patients all underwent thyroid surgery in Shaanxi Provincial Cancer Hospital Affiliated to Medical School (Xi'an, Shaanxi, China). The diagnoses of 50 TMC tumors and 30 benign lesions were confirmed by pathological examination. The patients consisted of 21 men and 52 women aged 19-61 years (mean age, $39.5 \pm 10.3$ years). All patients underwent pre-operative contrast examination with CEUS and UE based on conventional ultrasonography. The present study was conducted in accordance with the Declaration of Helsinki (17) and with approval from the Ethics Committee of Xi'an Jiaotong University (Xi'an, China). Written informed consent was obtained from all participants.

Instruments and reagents. The Philips iU22 xMATRIX ultrasonography diagnostic apparatus and L9-3 probe (Koninklijke Philips N.V., Amsterdam, Netherlands) were used to select the thyroid examination conditions. The angiographic region of interest was defined as thyroid nodules with surrounding healthy tissue. The inclusion criterion was the presence of suspicious solid nodules without a background of diffused thyroid lesions. The instrument was equipped with UE and CEUS analysis software, with the mechanical index set at 0.06-0.08. The contrast agent used was SonoVue, which consisted of sulfur hexafluoride (SF6) packaged by lipid membrane (Bracco, Milan, Italy). Prior to use, $59 \mathrm{mg}$ dry lyophilized SonoVue powder was added to $5 \mathrm{ml}$ normal saline and repeatedly agitated to form the white SF6 microbubble suspension.

Ultrasonography. The patients were placed in the supine position, and conventional 2-dimensional and color Doppler ultrasonography of the thyroid were performed for all patients. If suspicious nodules were identified, the patient underwent qualitative evaluation of the lesions in accordance with the benign and malignant lesion differentiation standards proposed by Stacul et al (18).

Thyroid CEUS was conducted using the dual-contrast mode and by adjusting the gain to only capture the thyroid tunica. Additionally, the single focus was set at the lower level of the lesion. Subsequent to noting the name, age and sex of each patient and ensuring that the setting parameters were correct, the position of the probe was fixed to prevent slippage. Next, the long axis view was selected. The mode was switched to ultrasonography contrast mode and a venous channel was created using a 20-ml trocar to puncture the superficial elbow vein. Following agitation and blending for the rapid bolus injection, $2.4 \mathrm{ml}$ contrast agent was intravenously injected prior to washing the pipe with $5.0 \mathrm{ml}$ saline. Simultaneously, and maintaining same observation view, the timer was started. This allowed real-time observation of the dynamic images to be performed exactly 3 min after administration of the contrast agent. The recorded observations were stored on the built-in hard disc for subsequent off-line analysis, with the imaging data coded according to a random order. The ultrasonography results included the size, location and shape of the radiographic lesion. These data were confirmed by a surgeon and a pathologist to ensure that the intraoperative lesion corresponded with the nodules observed during contrast-enhanced ultrasonography and pathological examination.

Following adjustment of the position and size of the sampling frame to ensure that the lesions and surrounding healthy tissue were contained, UE was conducted using the dual mode for observation in the vertical section. The probe was vertically vibrated using light pressure according to the indicator to ensure that the pressure index remained constant between 3 and 4 for 3-4 s. Subsequently, the color change of the sampling frame was observed and hardness scores were calculated for each lesion. Different colors represent the elasticity and hardness of the tissue. Red represents soft tissue, green represents moderate hard tissue, and blue represents inelastic hard tissue; if $>50 \%$ of the lesion area was green, the lesion was considered predominantly green, and vice versa for blue. All patients were examined by 2 senior clinicians specialized in contrast-enhanced ultrasonography.

Result evaluation. A double blind method was used for the 2 examinations and evaluation methods. Prior to thorough discussion of each case, the CEUS and UE findings were confirmed by 2 experienced ultrasonography specialists and diagnoses were determined. The following 5-point method was used to analyze the UE data: If the cystic lesions exhibited blue and green or red-green-blue coloring, the score was 0 points; if the lesions and the surrounding tissue demonstrated homogeneous green coloring, the score was 1 point; if the lesion area was $>90 \%$ green, the score was 2 points; if the lesion exhibited no incidences of blue and green, and predominantly blue (60-90\%), the score was 3 points; and if $>90 \%$ of the lesion was blue, the score was 4 points. If the lesion scored $\geq 3$ points, the lesion was diagnosed as a malignant tumor, whereas if the lesion scored $\leq 2$ points, it was diagnosed as a benign tumor (19). CEUS evaluation was performed by initially comparing the enhancement degree of the lesion and surrounding healthy tissue. Enhancement was categorized into light and weak (low enhancement), equal and high enhancement, according to the difference between nodules and surrounding tissues, judged by eye. Next, the enhancement mode (homogeneous degree) was divided into homogeneous and inhomogeneous enhancement. The phenomenon of enhancement decreasing below surrounding tissues quickly was defined as early regression. Inhomogeneous perfusion and whole course low enhancement were considered to be the diagnostic indices for malignant nodules $(12,18)$.

Statistical analysis. The accuracy of the 2 diagnostic methods was calculated using SPSS statistical software, version 13.0 (SPSS, Inc., Chicago, IL, USA). Comparisons between the sensitivity, specificity and accuracy rates were 


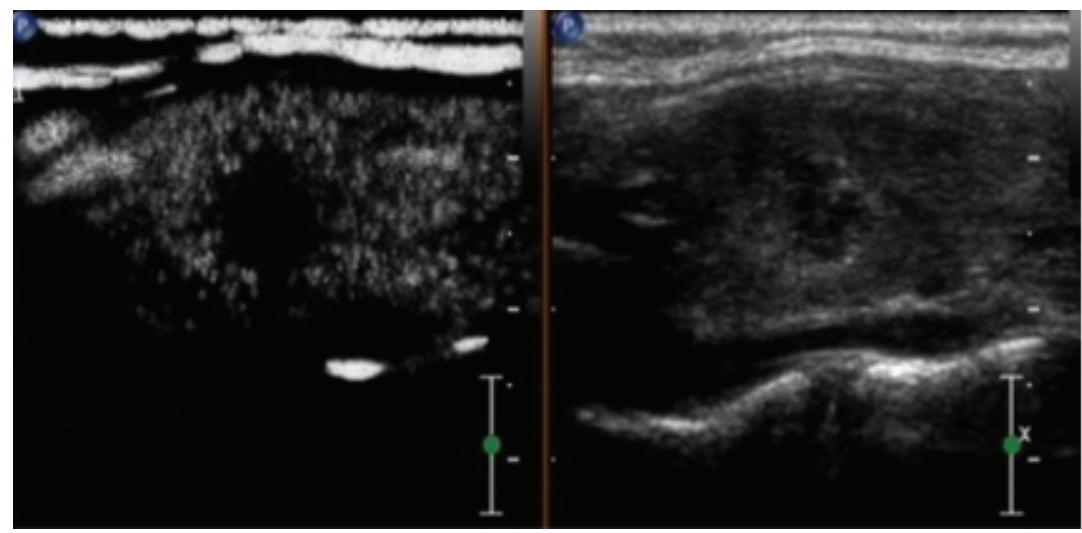

Figure 1. Ultrasound contrast imaging of thyroid microcarcinoma nodules indicating a lack of good blood supply, and therefore low perfusion. The ultrasonic appearance was consistent with the pathological findings.

A

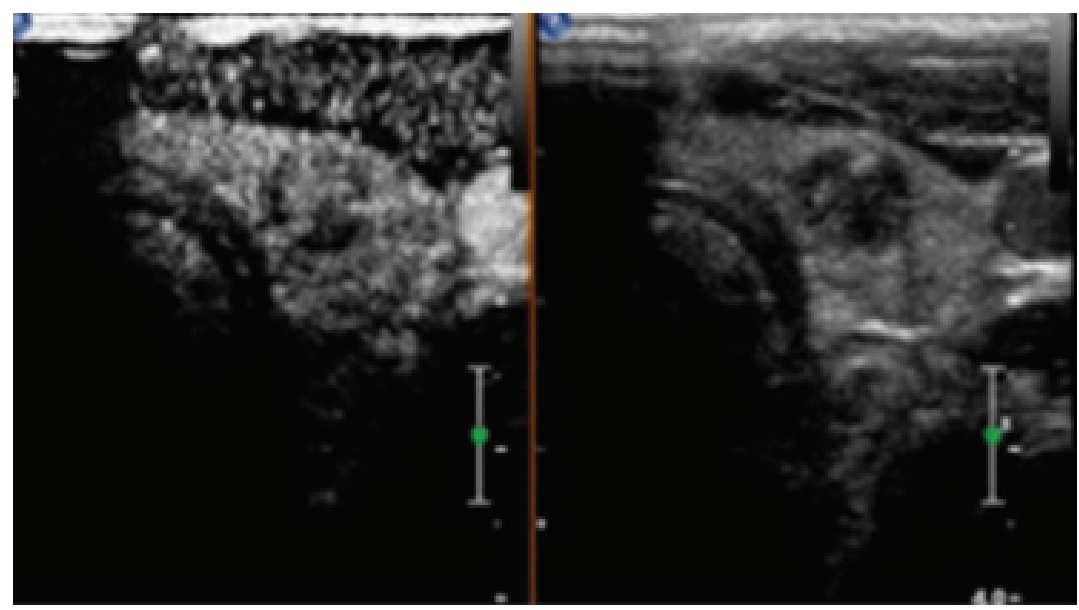

B

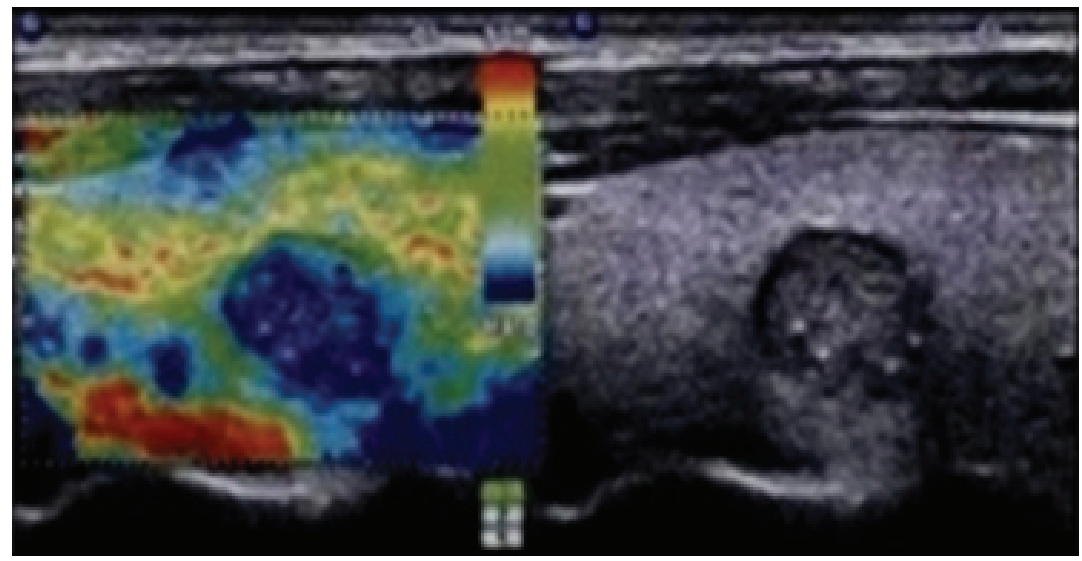

Figure 2. (A and B) Comparison between contrast-enhanced ultrasound (CEUS) and ultrasonic elastography (UE) images of thyroid microcarcinoma nodules in a single patient. (A) CEUS cross-section demonstrating weak enhancement of the nodule. The ultrasonic indication was that the nodular nature was to be determined. (B) Longitudinal UE section demonstrating $>90 \%$ blue coloring of the nodule (score, 4 points). The coloring indicates a malignant lesion, consistent with the pathological findings.

analyzed using the $\chi^{2}$ test and $\mathrm{P}<0.05$ was considered to indicate a statistically significant difference.

\section{Results}

CEUS features. The CEUS features of the 80 thyroid micronodules are indicated in Table I. With regards to enhancement degree, 10 benign nodules and 31 malignant nodules presented a low degree; 14 benign and 15 malignant nodules presented an equal degree; and 6 benign and 4 malignant nodules presented a high degree. A total of 22 benign and 37 malignant nodules presented homogeneous enhancement, while 8 benign and 13 malignant nodules displayed no homogeneous enhancement. A total of 6 benign and 18 malignant nodules exhibited early regression, while the remaining nodules did not. The CEUS indices exhibited no 
Table I. Comparison of contrast-enhanced ultrasound enhancement modes between the benign and malignant thyroid micronodules (diameter, $\leq 10 \mathrm{~mm} ; \mathrm{n}=80)^{\mathrm{a}}$.

\begin{tabular}{|c|c|c|c|c|c|c|c|}
\hline \multirow[b]{2}{*}{ Diagnosis } & \multicolumn{3}{|c|}{ Enhancement degree } & \multicolumn{2}{|c|}{$\begin{array}{l}\text { Homogeneous } \\
\text { enhancement }\end{array}$} & \multicolumn{2}{|c|}{ Early regression } \\
\hline & Low & Equal & High & Yes & No & Yes & No \\
\hline Benign & 10 & 14 & 6 & 22 & 8 & 6 & 24 \\
\hline Thyroid microcarcinoma & 31 & 15 & 4 & 37 & 13 & 18 & 32 \\
\hline
\end{tabular}

${ }^{a}$ No statistical differences was identified between the benign and malignant nodules $(\mathrm{P}>0.05)$.

Table II. Results of CEUS and UE in the examination of thyroid microlesions.

\begin{tabular}{lcrccccc}
\hline & & \multicolumn{2}{c}{ Pathological diagnosis } & & & \\
\cline { 3 - 4 } Examination method & Imaging diagnosis & TMC & Benign & & Sensitivity, \% & Specificity, \% & Accuracy, \% \\
\hline \multirow{2}{*}{ CEUS } & TMC & 44 & 6 & 88.0 & 80.0 & 85.0 \\
UE & Benign & 6 & 24 & & $94.0^{\mathrm{a}}$ & $90.0^{\mathrm{a}}$ & $92.5^{\mathrm{a}}$ \\
& TMC & 47 & 3 & & & \\
\hline
\end{tabular}

${ }^{\mathrm{a}} \mathrm{P}<0.05$ vs. corresponding CEUS results. CEUS, contrast-enhanced ultrasound; UE, ultrasonic elastography; TMC, thyroid microcarcinoma.

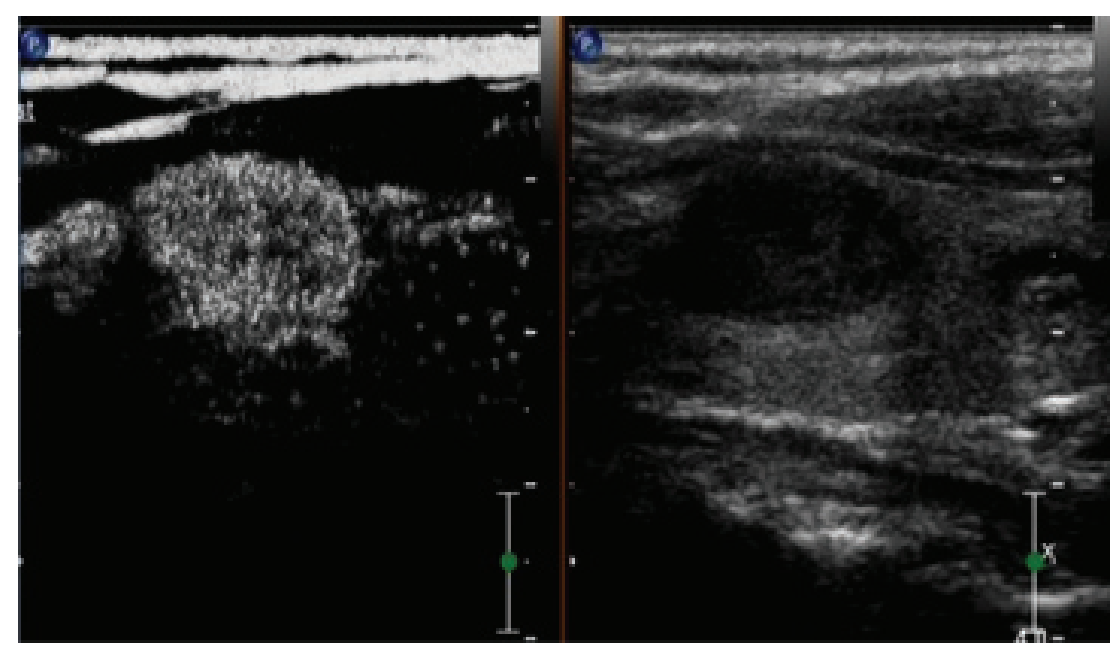

Figure 3. Contrast-enhanced ultrasound of a nodular goiter demonstrating uniform high enhancement.

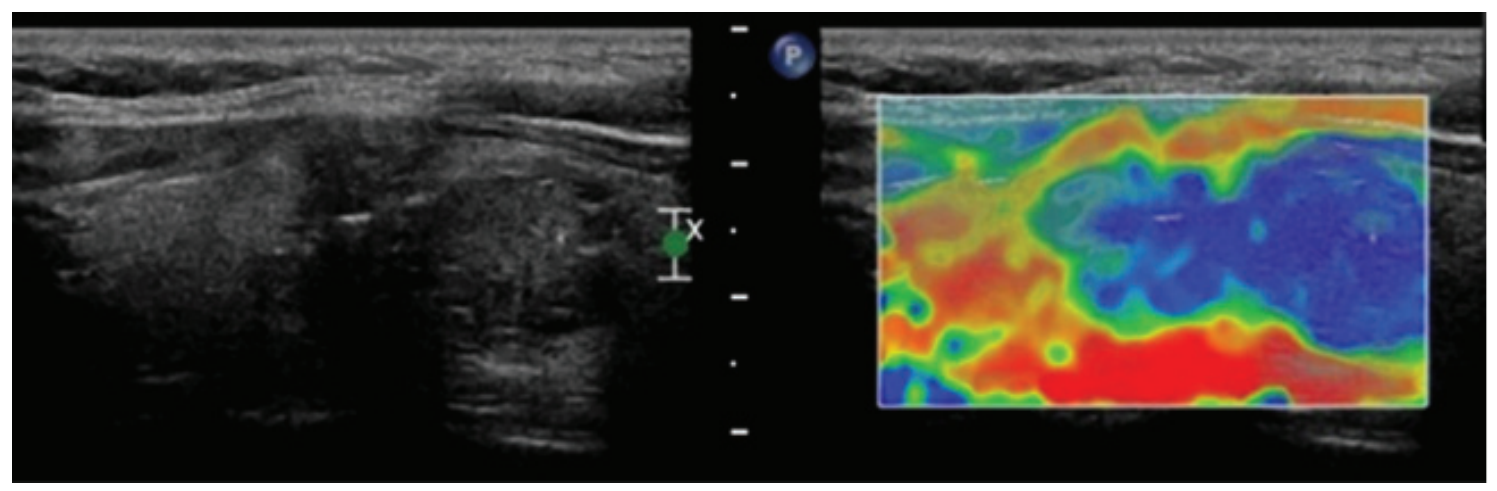

Figure 4. Ultrasonic elastography of a thyroid microcarcinoma, resulting in a score of 4 points, which was consistent with the pathological findings. 
significant differences between benign and malignant thyroid nodules (diameter, $\leq 10 \mathrm{~mm} ; \mathrm{P}>0.05$ ), indicating that CEUS has no evident advantage in the diagnosis of TMC.

Comparison of CEUS and UE. The consistency and accuracy of CEUS and UE for the diagnosis of thyroid micronodules are indicated in Table II. The results of the pathological diagnosis were considered as the gold standard for diagnosis. Accordingly, the correct diagnostic rate of CEUS was determined to be $85 \%$ (68/80 thyroid micronodules), out of which 6 benign cases were misdiagnosed as TMC and 6 cases of TMC were misdiagnosed as benign nodules. The correct diagnostic rate of the UE 5-point scoring method was $92.5 \%$ (74/80 cases), with 3 cases of TMC misdiagnosed as benign nodules and 3 cases of benign nodules misdiagnosed as TMC. An elasticity imaging score of $\geq 3$ points for the diagnosis of TMC was determined to have diagnostic sensitivity of $94.0 \%$, specificity of $90.0 \%$ and accuracy of $92.5 \%$; whereas the corresponding rates for CEUS were 88.0, 80.0 and $85.0 \%$, respectively. Thus, ultrasonographic elasticity imaging was determined to demonstrate more statistically significant advantages in the accurate diagnosis of TMC compared with CEUS $(\mathrm{P}<0.05)$.

Ultrasound Contrast Imaging. Four typical patients were selected for the representative images. The first patient was female and 26 years old, whose ultrasound contrast imaging of thyroid microcarcinoma nodules indicated a lack of good blood supply, and therefore low perfusion, and the ultrasonic appearance was consistent with the pathological findings (Fig. 1.) The second patient was female and 38 years old, whose CEUS cross-section demonstrated weak enhancement of the nodule. The ultrasonic indication was that the nodular nature was to be determined (Fig. 2A) Longitudinal UE section demonstrating $>90 \%$ blue coloring of the nodule (score, 4 points). The coloring indicates a malignant lesion, consistent with the pathological findings (Fig. 2B). The third patient was female and 61 years old whose contrast-enhanced ultrasound of a nodular goiter demonstrated uniform high enhancement (Fig. 3). The fourth patient was female and 61 years old whose ultrasonic elastography of a thyroid microcarcinoma, resulted in a score of 4 points, which was consistent with the pathological findings (Fig. 4).

\section{Discussion}

Currently, there is significant controversy regarding the application of CEUS in the diagnosis of thyroid diseases, with different studies reporting contradictory results on its value $(12,20)$. Zhang et al (12) reported that thyroid nodule-enhancing CEUS models may be divided into homogeneous, inhomogeneous (heterogeneous), ring (ring-enhancing) and no enhancement nodules. In addition, homogeneous enhancement was considered to be characteristic of benign tumors, with the sensitivity and specificity of heterogeneous low enhancement in the prediction of malignant thyroid tumors determined as 88.2 and $92.5 \%$, respectively. Furthermore, Bartolotta et al (21) reported that nodules measuring $<1 \mathrm{~cm}$ in diameter were predominantly characterized by poorly-vascularized features; nodules measuring $1-2 \mathrm{~cm}$ in diameter were characterized by a low level of speckled enhancement; and nodules measuring $>2 \mathrm{~cm}$ in diameter were characterized by a diffused enhanced performance. In the present study, CEUS identified poor vascularization, and a low (Fig. 1) or faint/weak (Fig. 2) enhancement degree in 31/50 TMC nodules (62\%). By contrast, the remaining 19 nodules $(38 \%)$ exhibited iso- or high enhancement, through high or full coverage of the lesions by the contrast agent, in the lesions and surrounding tissues when the contrast agent was injected. Among these 19 nodules, 6 were diagnosed as benign. Among the 30 pathologically confirmed benign nodules, 20 nodules $(66.66 \%)$ exhibited iso- or high enhancement (Fig. 3), which pre-operatively confirmed the diagnosis of benign nodules. However, the remaining 10 nodules $(33.4 \%)$ exhibited low or weak enhancement on CEUS. Among these, 6 nodules were misdiagnosed as TMC. Fine-needle puncture biopsies were subsequently performed to diagnose these nodules as 1 granulomatous nodule, 1 case of old blood clot and 4 hyperplastic nodules with calcification. Thus, in contrast to the findings of Bartolotta et al (21), the present study determined that benign nodules measuring $<1 \mathrm{~cm}$ in diameter and malignant thyroid nodules exhibit numerous overlapping features on CEUS.

The results of the present study indicate that CEUS has no significant advantage in the identification of TMCs. The current authors hypothesize that the observed overlap in CEUS perfusion was a result of the specific tissue biostructures of the investigated nodules. Jebreel et al (22) reported that differences in the microvessel density of benign lesions measuring $<1 \mathrm{~cm}$ in diameter and malignant nodules may not be evident when the tumors are small. Furthermore, small malignant nodules may not exhibit typical characteristics of malignant tumors, including a large variation in vascular diameter and shape, a large number of vascular branches, easy formation of arteriovenous fistulae, low efficiency of new tumor vessels, or edema and fibrosis of the lesion stroma (22). In addition, the overlapping may be associated with the instrument sensitivity, and the adjustments and parameters employed during use of the instrument.

UE was originally reported by Ophir et al (23) in 1991, and is based on the interpretation of amplitude changes of echo signals prior to and following compression of the tissue. The amplitude changes are transferred into real-time color images, where the color elasticity coding of various tissues reflects the tissue hardness (24). A long-term clinical study determined that the hardness of lesions differs between malignant and benign tissues, with increased hardness being associated with an increased risk of malignancy (25). Furthermore, a study conducted by Wang et al (26) identified that UE had high diagnostic sensitivity and specificity for nodules measuring $<1 \mathrm{~mm}$ in diameter. Similarly, the sensitivity, specificity and accuracy of UE diagnosis of the 80 TMC cases in the present study (Fig. 4) were 94.0, 90.0 and 92.5\%, respectively. These results were significantly increased compared with the corresponding values obtained by CEUS $(\mathrm{P}<0.05)$.

The current authors propose that the advantages of UE in diagnosing TMC compared with CEUS may be due to the close association of the TMC nodule with the surrounding tissues. Thus, the corresponding deformation required may have been small. Furthermore, the nodules were commonly accompanied by psammoma bodies, resulting in the lesions 
exhibiting a certain level hardness and consequently a higher elasticity score, thus, improving the diagnostic accuracy rate of UE for TMC (27).

However, the elastic coefficients of different pathological tissues may overlap (28), indicating that UE is associated with various influencing factors that may result in misdiagnosis. Accordingly, 3/50 TMC nodules were misdiagnosed in the present study due to elastic scores of $\leq 2$ points, including 2 cases of follicular carcinoma. We hypothesize that the reason for this misdiagnosis may be that the investigated lesions were composed of various degrees of differentiated follicular tissue. For example, the stroma is rich in thin-walled vasculature and the blood flow is more evident in these lesions compared with in papillary carcinoma (29). If follicular differentiation is good, the hardness does not markedly differ from that of healthy thyroid tissue, resulting in a low elastic classification (29). In the present study, the other misdiagnosed lesion was in a patient with Hashimoto's disease accompanied by small papillary carcinoma. This lesion was likely misdiagnosed due to the increasing hardness of the thyroid caused by extensive damage to the thyroid parenchyma and tissue fibrosis hyperplasia, resulting in hardness differences between the lesions and a reduction in the size of peripheral tissues, thereby resulting in a reduced nodular elastic classification. A total of 3 nodules in the benign group had an elastic score of 4 points, 1 of which was diagnosed as a cystic adenoma. Due to the increase in liquid tension in the cystic sac, the true hardness of internal solid nodule was not accurately reflected, resulting in increased hardness and a higher score, according to the elasticity imaging 5-point diagnostic method. Furthermore, the current cohort included 1 case of subacute thyroiditis and 1 case of Hashimoto's thyroiditis, in which the nodules appeared hard due to repeated stimulation of inflammation, fibrosis, hyalinization and other pathological changes. Additionally, the hardness of the thyroid gland itself may affect the thyroid nodules in elasticity imaging (30).

According to the literature (31), 20-50\% of all TMC cases may be associated with cervical lymph node metastasis. Lyshchik et al (32) and Alam et al (33) reported that UE may improve the accuracy of the differential diagnosis of cervical lymph node metastasis. The results of the present study require exploration in future large-scale studies to clarify these findings.

In conclusion, it was determined that there was no significant advantage of using CEUS in the diagnosis of TMC. However, the novel technology of UE was demonstrated to be superior to conventional ultrasonography in evaluating the tissue biomechanics of TMC, and may provide relevant and useful data regarding tissue hardness, expanding the use of ultrasonography for diagnosis. Furthermore, the current results indicate a potential role of UE and hardness testing in the diagnosis of small papillary thyroid nodules.

\section{Acknowledgements}

The present study was supported by the Social Development Research Plan Project of Shaanxi Provincial Department of Science and Technology (grant nos. 2012K13-02-39 and 2011k15-06-14).

\section{References}

1. Müller $\mathrm{O}$ and Krawinkel M: Malnutrition and health in developing countries. CMAJ 173: 279-286, 2005.

2. Golden SH, Robinson KA, Saldanha I, Anton B and Ladenson PW: Clinical review: Prevalence and incidence of endocrine and metabolic disorders in the United States: A comprehensive review. J Clin Endocrinol Metab 94: 1853-1878, 2009.

3. Roti E, degli Uberti EC, Bondanelli $\mathrm{M}$ and Braverman LE: Thyroid papillary microcarcinoma: A descriptive and meta-analysis study. Eur J Endocrinol 159: 659-673, 2008.

4. Wong CK and Wheeler MH: Thyroid nodules: rational management. World J Surg 24: 934-941, 2000.

5. Haber RS: Role of ultrasonography in the diagnosis and management of thyroid cancer. Endocr Pract 6: 396-400, 2000.

6. Guth S, Theune U, Aberle J, Galach A and Bamberger CM: Very high prevalence of thyroid nodules detected by high frequency (13 MHz) ultrasound examination. Eur J Clin Invest 39: 699-706, 2009.

7. Isidro ML, Penín M and Cordido F: Systematic ultrasound evaluation of thyroid nodules. Thyroid 16: 1324-1325, 2006.

8. RotiE, degli UbertiEC, Bondanelli Mand Braverman LE: Thyroid papillary microcarcinoma: a descriptive and meta-analysis study. Eur J Endocrinol 159: 659-673, 2008.

9. Holtel MR: Emerging technology in head and neck ultrasonography. Otolaryngol Clin North Am 43: 1267-1274, 2010.

10. Yu D, Han Y and Chen T: Contrast-enhanced ultrasound for differentiation of benign and malignant thyroid lesions Meta-analysis. Otolaryngol Head Neck Surg 151: 909-915, 2014.

11. Carneiro-Pla D: Ultrasound elastography in the evaluation of thyroid nodules for thyroid cancer. Curr Opin Oncol 25: 1-5, 2013.

12. Zhang B, Jiang YX, Liu JB, Yang M, Dai Q, Zhu QL and Gao P: Utility of contrast-enhanced ultrasound for evaluation of thyroid nodules. Thyroid 20: 51-57, 2010.

13. Giuseppetti GM, Martegani A, Di Cioccio B and Baldassarre S: Elastosonography in the diagnosis of the nodular breast lesions: preliminary report. Radiol Med 110: 69-76, 2005.

14. Luo S, Kim EH, Dighe M and Kim Y: Thyroid nodule classification using ultrasound elastography via linear discriminant analysis. Ultrasonics 51: 425-431, 2011.

15. Ding J, Cheng H, Ning C, Huang J and Zhang Y: Quantitative measurement for Thyroid cancer characterization based on elastography. J Ultrasound Med 30: 1259-1266, 2011.

16. Bhatia KS, Rasalkar DP, Lee YP, Wong KT, King AD, Yuen HY and Ahuja AT: Cystic change in change in thyroid nodules: A confounding factor for real-time qualitative thyroid ultrasound elastography. Clin Radiol 66: 799-807, 2011.

17. Rickham PP: Human experimentation. Code of ethics of the World Medical Association. Declaration of Helsinki. Br Med J 2: $177,1964$.

18. Stacul F, Bertolotto M, De Gobbis F, et al: US, colour-Doppler US and fine-needle aspiration biopsy in the diagnosis of thyroid nodules. Radiol Med 11: 751-762, 2007.

19. Rago T, Santini F, Scutari M,Pinchera A and Vitti P: Elastography: new developments in ultrasound for predicting malignancy in thyroid nodules. J Clin Endocrinol Metab 92: 2917-2922, 2007.

20. Friedrich-Rust M, Sperber A, Holzer K, et al: Real-time elastography and contrast-enhanced ultrasound for the assessment of thyroid nodules. Exp Clin Endocrinol Diabetes 118: 602-609, 2010.

21. Bartolotta TV, Midiri M, Galia M, et al: Qualitative and quantitative evaluation of solitary thyroid nodules wih contrast-enhanced ultrasound: initial results. Eur Radiol 16: 2234-2241, 2006.

22. Jebreel A, England J, Bedford K, Murphy J, Karsai L and Atkin S: Vascular endothelial growth factor (VEGF), VEGF receptors expression and microvascular density in benign and malignant thyroid diseases. Int J Exp Pathol 88: 271-277, 2007.

23. Ophir J, Céspedes I, Ponnekanti H, Yazdi Y and Li X: Elastography: a quantitative method for imaging the elasticity of biological tissues. Ultrason Imaging 13: 111-134, 1991.

24. Khalil AS, Chan RC, Chau AH, Bouma BE and Mofrad MR: Tissue elasticity estimation with optical coherence elastography: toward mechanical characterization of in vivo soft tissue. Ann Biomed Eng 33: 1631-1639, 2005.

25. Pacini F, Schlumberger M, Dralle H, Elisei R, Smit JW and Wiersinga W; European Thyroid Cancer Taskforce: European consensus for the management of patients with differentiated thyroid carcinoma of the follicular epithelium. Eur J Endocrinol 154: 787-803, 2006. 
26. Wang Y, Dan HJ, Dan HY, Li T and Hu B: Differential diagnosis of small single solid thyroid nodules using real-time ultrasound elastography. Int Med Res 38: 466-472, 2010.

27. Lyshchik A, Higashi T, Asato R, et al: Thyroid gland tumor diagnosis at US elastography. Radiology 237: 202-211, 2005.

28. Hiltawsky KM, Krüger M, Starke C, Heuser L, Ermert H and Jensen A: Freehand ultrasound elastography of breast lesions: clinical results. Ultrasound Med Biol 27: 1461-1469, 2001.

29. Ren X, Zhan W and Zhou P: Comparative study of real-time elastography and grey-scale ultrasonography in the diagnosis of thyroid occupied lesions. Chin J Ultrasound Med 25: 128-132, 2009.

30. Shuzhen C: Comparison analysis between conventional ultrasonography and ultrasound elastography of thyroid nodules. Eur J Radiol 81: 1806-1811, 2012.
31. Costa S, Giugliano G, Santoro L, et al: Role of prophylactic central neck dissection in $\mathrm{cN} 0$ papillary thyroid cancer. Acta Otorhinolaryngol Ital 29: 61-69, 2009.

32. Lyshchik A, Higashi T, Asato R, et al: Cervical lymph node metastases: diagnosis at sonoelastography - initial experience. Radiology 243: 258-267, 2007.

33. Alam F, Naito K, Horiguchi J, Fukuda H, Tachikake T and Ito $\mathrm{K}$ : Accuracy of sonographic elastography in the differential diagnosis of enlarged cervical lymph nodes: comparison with conventional B-mode sonography. AJR Am J Roentgenol 191: 604-610, 2008. 